\section{SMGrøup}

SM

\section{Musculoskeletal Disorders}

\section{Article Information}

Received date: Jun 18, 2016

Accepted date: Aug 09, 2016

Published date: Aug 16, 2016

\section{Corresponding author}

Hiroshi Kawaguchi, The Chief of the Spine Center, Japan Community Health Care Organization ( $\mathrm{JCHO}$ ), Tsukudo 5-1, Tokyo 162-8543, Japan, Tel: 03-3269-8111; Fax: 03-3260-7840; Email: kawaguchi0126@gmail.com

Distributed under Creative Commons CC-BY 4.0

Keywords Osteoarthritis; Chondrocytes Hypertrophy; Apoptosis; Endochondral ossification; Cartilage

Article DOI 10.36876/smmd.1003

\title{
Endochondral Ossification Signals May be Important for the Osteoarthritis Progression
}

\author{
Hiroshi Kawaguchi \\ The Chief of the Spine Center, Japan Community Health Care Organization (JCHO), Tokyo, Japan '
}

\section{Abstract}

Osteoarthritis $(\mathrm{OA})$, one of the most common skeletal disorders characterized by cartilage degradation and osteophyte formation in joints, is induced by accumulated mechanical stress; however, little is known about the underlying molecular mechanism. Several experimental OA models in mice by producing instability in the knee joints have been developed to apply approaches from mouse genetics. Although proteinases like matrix metalloproteases and aggrecanases have now been proven to be the principal initiators of OA progression, clinical trials of proteinase inhibitors have not been successful for the treatment, turning the interest of researchers to the upstream signals of proteinase induction. These signals include under graded and fragmented matrix proteins like type II collagen or fibronection that affects chondrocytes through distinct receptors. Another signal is proinflammatory factors that are produced by chondrocytes and synovial cells; however, recent studies that used mouse OA models in knockout mice did not support that these factors have a role in the central contribution to OA development. Our mouse genetic approaches found that the induction of a transcriptional activator Runx2 in chondrocytes under mechanical stress contributes to the pathogenesis of OA through chondrocyte hypertrophy. In addition, chondrocyte apoptosis has recently been identified as being involved in OA progression. We hereby propose that these endochondral ossification signals may be important for the OA progression, suggesting that the related molecules can clinically be therapeutic targets of this disease.

\section{Introduction}

OA, which affects all joints of the body, is characterized by two aspects: one is cartilage degradation shown as a joint space narrowing on radiographs, and the other is osteophyte formation at the edge of the joints (Figure 1). Despite significant social demand for more information, risk factors of this disease identified by epidemiologic studies have to date been limited to age, obesity, trauma history, occupation, and gender [1]. These factors are closely related to the accumulation of mechanical stress to joints (Figure 1).

In an effort to clarify the mechanisms whereby the mechanical stress leads to OA development, experimental animal models in which joint instability is induced through surgical intervention have been developed in dogs, rabbits, guinea pigs and rats [2-8]. Considering that mouse is now the most ideal animal for the molecular study due to recent progress in mouse genetics and the availability of transgenic and knockout mice, we and others have established mechanical instability-induced OA models in mice that are reproducible and resemble human $\mathrm{OA}$, using a microsurgical technique to produce instability in the knee joints. Most popular is the model developed by Glasson's group which involves Destabilization of the Medial Meniscus (DMM) by transection of meniscotibial ligament anchoring the medial meniscus to the tibial plateau [9]. We have developed the medial model by resection of the medial meniscus and medial colateral ligament [10]. In addition, there is a traditional Anterior Cruciate Ligament Transection (ACLT) model that has also been used in larger animals [11].

\section{Proteinases for OA Induction}

Normal joint cartilage is constituted of a framework of type II collagen (COL2), in which proteoglycan connected to hyaluronic acid waves smoothly. Because it contains a large quantity of water, proteoglycan provides elasticity and lubricity to the joint surface. However, in the OA cartilage, most of the proteoglycans are cut, fragmented, and floating in the synovial fluid. Due to the loss of the shock-absorber, the mechanical stress is loaded directly onto COL2, which is also cut and degraded. It has been shown that the initiation of OA occurs with the appearance of proteinases such as Matrix Metalloproteinases (MMPs) and aggrecanases that sever the core proteins of proteoglycans at their specific cleavage sites [12]. Actually, the importance of a proteinase, ADAMTS5, also called aggrecanase-2, was proved by back-to-back publications in 2005 $[13,14]$, creating a DMM model in knockout mice which showed resistance to cartilage degradation under the OA induction. However, the clinical trials of the proteinase inhibitors for the OA treatment have not been successful due to side effects like musculoskeletal pain, tendinitis, and so

\section{ISSN: 2576-5442}




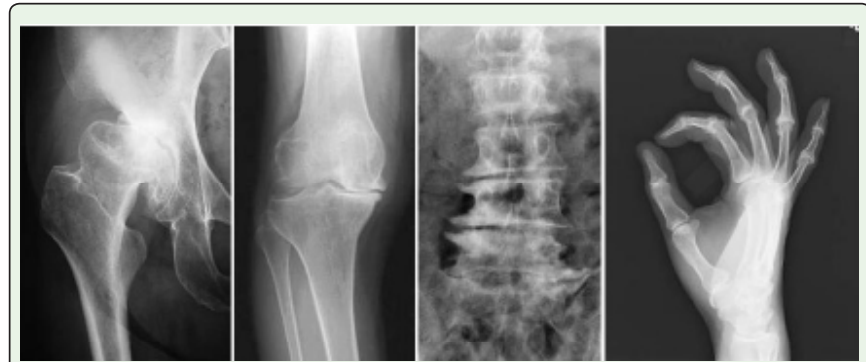

Figure 1: Radiographs of Osteoarthritis (OA) in hip, knee, lumbar spine, and hand. Cartilage degradation shown as joint space narrowing and osteophyte formation at the edge of the joints is two major disorders of OA.

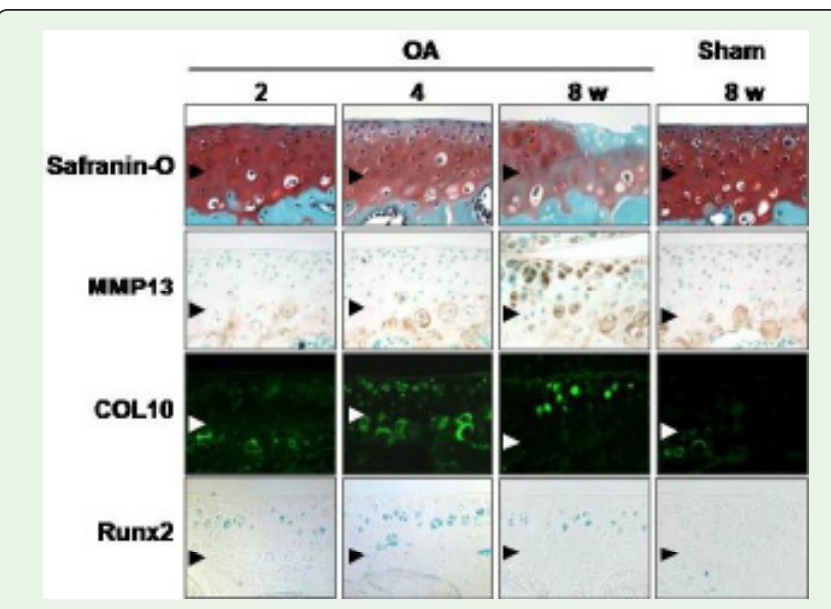

Figure 2: Time course of expressions of MMP-13, COL10, and Runx2 in the medial tibial cartilage of OA-induced and sham-operated knee joints in the mouse medial model. Localization of MMP-13 and COL 10 was detected by immunohistochemistry, and Runx2 localization was detected by X-gal staining of heterozygous Runx2 deficient mice with LacZ knock-in at the site of Runx2 deletion (Runx2+/lacz). Arrowheads indicate the level of tidemark.

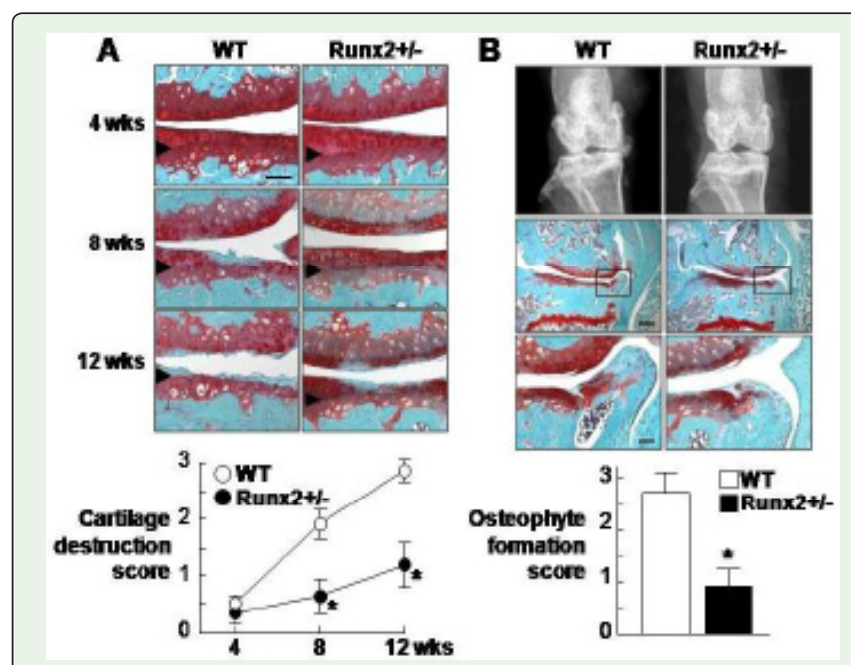

Figure 3: (A) Safranin-O staining and the cartilage degradation score of the medial tibial cartilage of Runx2-/-and wild-type (WT) littermates. Arrowheads indicate the level of tidemark. (B) Anteroposterior X-ray features, Safranin-O staining, and the osteophyte formation score 12 weeks after surgery. Means (symbols or bars) \pm SEM (error bars). ${ }^{*} \mathrm{P}<0.01$ vs. WT. on $[15,16]$. Hence, researchers are now turning their attention to the signals inducing the proteinases in chondrocytes. Olsen's group and others have shown that matrix protein, especially undegraded COL2, may initiate it through a receptor tyrosine kinase Discoidin Domain Receptor 2 (DDR-2) $[17,18]$. This causes the degradation of matrix proteins, and the product fragments of COL2 or fibronection then induce proteinases through respective integrins $\alpha 2 \beta 1$ and $\alpha 5 \beta 1$ [17].

\section{Pro-Inflammatory Factors and OA}

Besides these matrix proteins, pro-inflammatry factors like Prostaglandins (PGs), Tumor Necrosis Factor- $\alpha$ (TNF- $\alpha$ ), Interleukin-1 (IL-1), IL-6, and nitric oxides are reported to induce proteinases through their respective receptors [19]. These proinflammatory factors are also reported to be produced by synovial cells under the stimulation of the under graded or fragmented matrix proteins [17]. However, it is suspected that these cytokines play significant roles in the OA development. Our previous report showed that levels of TNF- $\alpha$, IL-1, IL- 6 as well as fibroblast growth factor- 2 in the synovial fluid from knee joints of OA patients were much lower than those of rheumatoid arthritis patients [20]. Furthermore, a previous report using a mouse ACLT model showed that mice lacking IL-1, IL-1-converting enzyme, stromelysin 1 or inducible nitric oxide synthase unexpectedly exhibited an acceleration of cartilage degradation, implying that these pro-inflammatory factors do not stimulate, but rather inhibit such degradation [11].

PGE2, a representative pro-inflammatory factor, is also produced more abundantly in the OA cartilage than in normal cartilage [21], and microsomal PGE synthase-1 (mPGES-1) is a terminal enzyme for the PGE2 production in chondrocytes of OA patients [22]. Although we created the medial OA model in the PGES-1 knockout mice, the cartilage degradation and osteophyte formation were comparable to the will-type littemates [23]. We therefore believe that inflammation may be associated with the OA process as a consequence, but might not have a central role in the cause of OA initiation or progression.

\section{Chondrocyte Hypertrophy and OA}

Our examination of the time course of histology of the mouse joint cartilage using the medial OA model revealed that type $\mathrm{X}$ Collagen (COL10) and MMP-13 were significantly induced during OA progression (Figure 2) $[10,24]$. COL10 expression, a specific marker of hypertrophic chondrocytes, appeared in the superficial and middle zones above the tidemark at 4 weeks, consistent with previous studies $[25,26]$. MMP-13 expression appeared in the hypertrophic chondrocytes above the tidemark at 8 weeks. These findings suggest that articular chondrocytes undergo hypertrophic differentiation in response to joint instability, and the hypertrophic chondrocytes express MMP-13 that may degrade the cartilage matrix.

Since a transcriptional activator Runx 2 has been known to induce both chondrocyte hypertrophy and MMP-13 expression [27-29], we then examined the involvement of Runx2 during OA development [24]. Runx 2 expression was induced above the tidemark in the cartilage as early as 2 weeks, enhanced at 4 weeks, and decreased thereafter by the OA induction, which was not observed in the sham-operated cartilage (Figure 3). For the functional analyses of Runx2, we used heterozygous Runx2-knockout mice (Runx2+/-), since homozygous Runx2-knockout (Runx2-/-) mice died just after birth. The Runx2+/- mice showed normal skeletal development and 


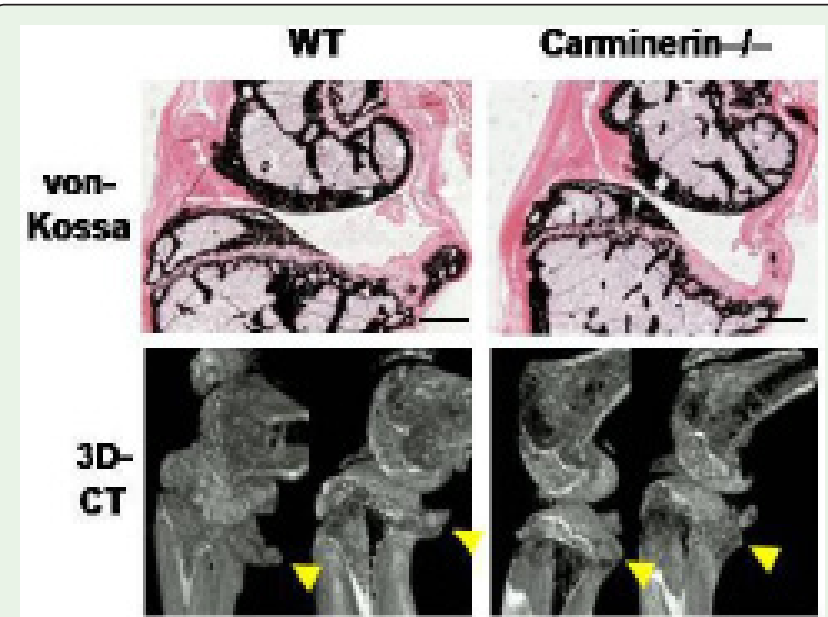

Figure 4: von Kossa staining and three-dimensional computed tomography (3D-CT) of knee joints of carminerin-/- and wild-type (WT) littermates in the ACLT model. OA was induced at the posterior tibias of the knee joint of 8-week-old mice and examined 10 weeks after surgery.

articular cartilage under physiological conditions. Both COL10 and MMP-13 expressions were decreased by the Runx2 insufficiency (data not shown), indicating that chondrocyte hypertrophy and MMP-13 induction during OA progression is at least partly mediated by Runx2. When the OA progression was compared between WT and Runx2+/- joints, the cartilage degradation in Runx2+/- was much milder than that of the WT cartilage at 8 weeks and thereafter (Figure 3A). The Runx2+/- joint also showed decreased osteophyte formation (Figure 3B). These findings demonstrate that Runx2 contributes to cartilage degradation and the subsequent osteophyte formation under joint instability.

Our differential display analysis identified a novel molecule that was up-regulated by a high phosphate diet in association with calcification of auricular cartilage in mice, and we called it carminerin $[30,31]$. The knockout (carminerin -/-) mice did not show skeletal abnormality under physiological conditions. When we created the ACLT OA model, the joint destruction normally occurred; however, osteophyte formation seemed to be decreased by the carminerin knockout, indicating that carminerin is not essential for cartilage degradation but plays a role in the osteophyte formation (Figure 4) [32].

\section{Discussion}

In addition to hypertrophic differentiation of chondrocytes, chondrocyte apoptosis is known to be involved in OA development [33]. Intraarticular injection of a pan-caspase inhibitor has been reported to suppress cartilage degradation under OA induction in a mouse ACLT model [34]. When we created the OA medial model in the hetero knockout mice of osteoprotegerin that is well known as a decoy receptor of RANKL for osteoclastic bone resorption, joint destruction was enhanced as compared to the wild-type littermates [35]. On the contrary, an intraarticular injection of recombinant osteoprotegerin suppressed joint destruction with a decrease of apoptotic chondrocytes. Since Tumor Necrosis Factor-Related Apoptosis-Inducing Ligand (TRAIL), a ligand of osteoprotegerin, induces chondrocyte apoptosis [36,37], osteoprotegerin might inhibit the apoptosis induced by TRAIL. These in vivo findings

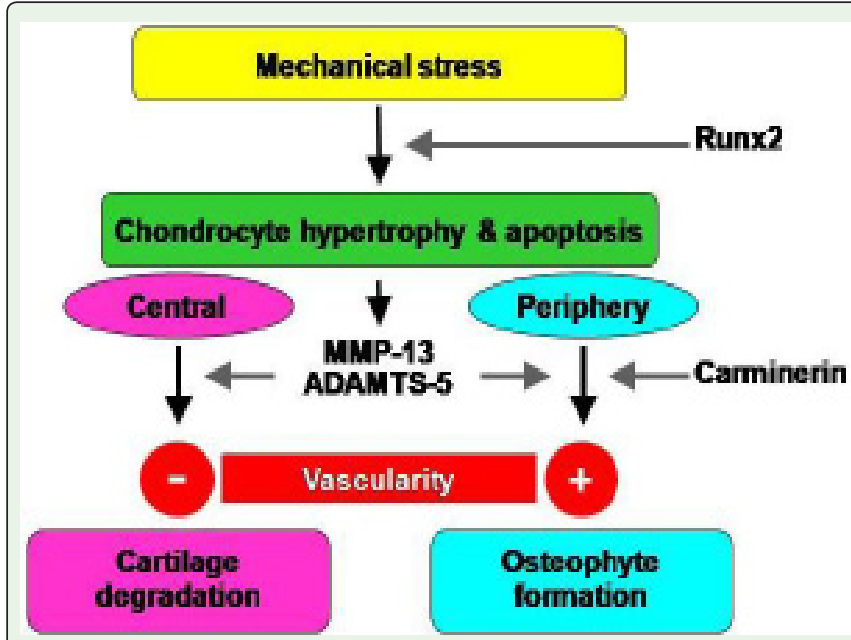

Figure 5: Molecular background of $\mathrm{OA}$ progression under mechanical stress by mouse genetics approaches.

clearly demonstrate that not only chondrocyte hypertrophy, but also chondrocyte apoptosis, both of which are signals of endochondral ossification, plays some roles in cartilage degradation during $\mathrm{OA}$ development under mechanical stress (Figure 5).

Figure 5 summarizes our hypothesis of the molecular background of $\mathrm{OA}$ progression under mechanical stress in joints. As the mechanism involved in this stress causing protease production, in addition to matrix proteins and pro-inflammatory signals, we hereby propose the importance of chondrocyte hypertrophy and apoptosis, which are signals for endochondral ossification. The proteinases produced by hypertrophic chondrocytes cause cartilage degradation at the center of the joint and osteophyte formation at the periphery. The difference of the two sites may depend on the vascularity. At the periphery, vascularity is accessible from synovium or tendon, which causes endochondroal ossification to occur and make osteophytes, just like at the growth plate. Carminerin may play a role in the chondrocyte calcification at this stage. However, in the center, the vascularity is not accessible from the edge, so that it ends up with cartilage degradation without being replaced by bone.

\section{Conclusion}

The ultimate aim of our study is to identify the molecular targets for clinical treatment of OA. Although we primarily used mouse genetics approaches, we have attempted to confirm the reproducibility of the mouse findings in humans as well through human gene polymorphism or clinical biochemical studies. Among the molecules introduced in this review, there are some whose suppression ameliorated skeletal disorders under pathological conditions but did not affect physiological conditions, indicating that the treatment targeting these molecules may lead to an ideal treatment without side effects on physiological functions. In fact, clinical trials based on the present findings are currently being practically planned.

\section{References}

1. Sharma L. Osteoarthritis year in review 2015: clinical. Osteoarthritis Cartilage. 2016; 24: 36-48.

2. Pond MJ, Nuki G. Experimentally-induced osteoarthritis in the dog. Ann Rheum Dis. 1973; 32: 387-388. 
3. Matyas JR, Ehlers PF, Huang D, Adams ME. The early molecular natural history of experimental osteoarthritis. I. Progressive discoordinate expression of aggrecan and type II procollagen messenger RNA in the articular cartilage of adult animals. Arthritis Rheum. 1999; 42: 993-1002.

4. Le Graverand MP, Eggerer J, Vignon E, Otterness IG, Barclay L, Hart DA. Assessment of specific mRNA levels in cartilage regions in a lapine model of osteoarthritis. J Orthop Res. 2002; 20: 535-544.

5. Matyas JR, Huang D, Chung M, Adams ME. Regional quantification of cartilage type II collagen and aggrecan messenger RNA in joints with early experimental osteoarthritis. Arthritis Rheum. 2002; 46: 1536-1543.

6. Bluteau G, Gouttenoire J, Conrozier T, Mathieu P, Vignon E, Richard M, et al. Differential gene expression analysis in a rabbit model of osteoarthritis induced by anterior cruciate ligament ACL section. Biorheology. 2002; 39 . 247-258

7. Hayami T, Pickarski M, Wesolowski GA, McLane J, Bone A, Destefano J, et al. The role of subchondral bone remodeling in osteoarthritis, reduction of cartilage degeneration and prevention of osteophyte formation by alendronate in the rat anterior cruciate ligament transection model. Arthritis Rheum. 2004 50: 1193-1206.

8. Lorenz H, Wenz W, Ivancic M, Steck E, Richter W. Early and stable upregulation of collagen type II. collagen type I and YKL40 expression levels in cartilage during early experimental osteoarthritis occurs independent of joint location and histological grading. Arthritis Res Ther. 2005; 7: R156-165

9. Glasson SS. In vivo osteoarthritis target validation utilizing geneticallymodified mice. Curr Drug Targets. 2007; 8: 367-376.

10. Kamekura S, Hoshi K, Shimoaka T, Chung U, Chikuda H, Yamada T, et al. Osteoarthritis development in novel experimental mouse models induced by knee joint instability. Osteoarthritis Cartilage. 2005; 13: 632-641.

11. Clements KM, Price JS, Chambers MG, Visco DM, Poole AR, Mason RM Gene deletion of either interleukin-1beta, interleukin-1beta-converting enzyme, inducible nitric oxide synthase, or stromelysin 1 accelerates the development of knee osteoarthritis in mice after surgical transection of the medial collateral ligament and partial medial meniscectomy. Arthritis Rheum. 2003; 48: 3452-3463

12. Little CB, Meeker CT, Golub SB, Lawlor KE, Farmer PJ, Smith SM, et al. Blocking aggrecanase cleavage in the aggrecan interglobular domain abrogates cartilage erosion and promotes cartilage repair. J Clin Invest. 2007; 117: 1627-1636.

13. Glasson SS, Askew R, Sheppard B, Carito B, Blanchet T, Ma HL, et al Deletion of active ADAMTS5 prevents cartilage degradation in a murine model of osteoarthritis. Nature. 2005; 434: 644-648.

14. Stanton H, Rogerson FM, East CJ, Golub SB, Lawlor KE, Meeker CT, et al ADAMTS5 is the major aggrecanase in mouse cartilage in vivo and in vitro. Nature. 2005; 434: 648-652.

15. Nagase H, Visse R, Murphy G. Structure and function of matrix metalloproteinases and TIMPs. Cardiovasc Res. 2006; 69: 562-573.

16. Burrage PS, Brinckerhoff CE. Molecular targets in osteoarthritis: metalloproteinases and their inhibitors. Curr Drug Targets. 2007; 8: 293-303.

17. Li Y, Xu L, Olsen BR. Lessons from genetic forms of osteoarthritis for the pathogenesis of the disease. Osteoarthritis Cartilage. 2007; 15: 1101-1105.

18. Xu L, Peng H, Wu D, Hu K, Goldring MB, Olsen BR, et al. Activation of the discoidin domain receptor 2 induces expression of matrix metalloproteinase 13 associated with osteoarthritis in mice. J Biol Chem. 2005; 280: 548-555.

19. Vincenti MP, Brinckerhoff CE. Transcriptional regulation of collagenase (MMP-1, MMP-13) genes in arthritis: integration of complex signaling pathways for the recruitment of gene-specific transcription factors. Arthritis Res. 2002; 4: 157-164

20. Manabe N, Oda H, Nakamura K, Kuga Y, Uchida S, Kawaguchi H. Involvement of fibroblast growth factor-2 in joint destruction of rheumatoid arthritis patients. Rheumatology (Oxford). 1999; 38: 714-720.
21. Jacques C, Sautet A, Moldovan M, Thomas B, Humbert L, Berenbaum F Cyclooxygenase activity in chondrocytes from osteoarthritic and healthy cartilage. Rev Rhum Engl Ed. 1999; 66: 701-704.

22. Kojima F, Naraba H, Miyamoto S, Beppu M, Aoki H, Kawai S. Membraneassociated prostaglandin $\mathrm{E}$ synthase-1 is upregulated by proinflammatory cytokines in chondrocytes from patients with osteoarthritis. Arthritis Res Ther 2004; 6: R355-365.

23. Yamakawa K, Kamekura S, Kawamura N, Saegusa M, Kamei D, Murakam $\mathrm{M}$, et al. Association of microsomal prostaglandin $\mathrm{E}$ synthase 1 deficiency with impaired fracture healing, but not with bone loss or osteoarthritis, in mouse models of skeletal disorders. Arthritis Rheum. 2008; 58: 172-183.

24. Kamekura S, Kawasaki Y, Hoshi K, Shimoaka T, Chikuda H, Maruyama Z, et al. Contribution of runt-related transcription factor 2 to the pathogenesis of osteoarthritis in mice after induction of knee joint instability. Arthritis Rheum. 2006; 54: 2462-2470.

25. von der Mark K, Kirsch T, Nerlich A, Kuss A, Weseloh G, Gluckert K, et al. Type $X$ collagen synthesis in human osteoarthritic cartilage. Indication of chondrocyte hypertrophy. Arthritis Rheum. 1992; 35: 806-811.

26. Boos N, Nerlich AG, Wiest I, von der Mark K, Ganz R, Aebi M. Immunohistochemical analysis of type-X-collagen expression in osteoarthritis of the hip joint. J Orthop Res. 1999; 17: 495-502.

27. Ueta C, Iwamoto M, Kanatani N, Yoshida C, Liu Y, Enomoto-Iwamoto M, et al. Skeletal malformations caused by overexpression of Cbfa1 or its dominant negative form in chondrocytes. J Cell Biol. 2001; 153: 87-100.

28. Takeda S, Bonnamy JP, Owen MJ, Ducy P, Karsenty G. Continuous expression of Cbfa1 in nonhypertrophic chondrocytes uncovers its ability to induce hypertrophic chondrocyte differentiation and partially rescues Cbfa1deficient mice. Genes Dev. 2001; 15: 467-481.

29. Jimenez MJ, Balbin M, Lopez JM, Alvarez J, Komori T, Lopez-Otin C Collagenase 3 is a target of Cbfa1, a transcription factor of the runt gene family involved in bone formation. Mol Cell Biol. 1999; 19: 4431-4442.

30. Okawa A, Nakamura I, Goto S, Moriya H, Nakamura Y, Ikegawa S. Mutation in Npps in a mouse model of ossification of the posterior longitudinal ligament of the spine. Nat Genet. 1998; 19: 271-273.

31. Koshizuka Y, Yamada T, Hoshi K, Ogasawara T, Chung U, Kawano H, Nakamura $Y$, et al. Cystatin 10, a novel chondrocyte-specific protein, may promote the last steps of the chondrocyte differentiation pathway. J Biol Chem. 2003; 278: 48259-48266.

32. Yamada T, Kawano H, Koshizuka Y, Fukuda T, Yoshimura K, Kamekura S et al. Carminerin contributes to chondrocyte calcification during endochondral ossification. Nat Med. 2006; 12: 665-670.

33. Kühn K, D'Lima DD, Hashimoto S, Lotz M. Cell death in cartilage. Osteoarthritis Cartilage. 2004; 12: 1-16.

34. D'Lima D, Hermida J, Hashimoto S, Colwell C, Lotz M. Caspase inhibitors reduce severity of cartilage lesions in experimental osteoarthritis. Arthritis Rheum. 2006; 54: 1814-1821.

35. Shimizu S, Asou Y, Itoh S, Chung UI, Kawaguchi H, Shinomiya K, et al. Prevention of cartilage degradation with intraarticular osteoclastogenesis inhibitory factor/osteoprotegerin in a murine model of osteoarthritis. Arthritis Rheum. 2007; 56: 3358-3365.

36. Lee SW, Lee HJ, Chung WT, Choi SM, Rhyu SH, Kim DK, et al. TRAIL induces apoptosis of chondrocytes and influences the pathogenesis of experimentally induced rat osteoarthritis. Arthritis Rheum. 2004; 50: 534-542.

37. Pettersen I, Figenschau Y, Olsen E, Bakkelund W, Smedsröd B, Sveinbjörnsson B. Tumor necrosis factor-related apoptosis-inducing ligand induces apoptosis in human articular chondrocytes in vitro. Biochem Biophys Res Commun. 2002; 296: 671-676. 